\title{
Temperature Modulated Thermoelectric Gas Sensors
}

\author{
G. Hagen, N. Leupold, S. Wiegärtner, H. Wittmann, R. Moos \\ Department of Functional Materials, University of Bayreuth, 95440 Bayreuth, Germany \\ Functional.Materials@uni-bayreuth.de
}

\begin{abstract}
:
The output of a thermoelectric gas sensor is a voltage signal that is proportional to the exothermicity, generated during oxidation of reducing gases on a catalyst. A temperature difference between the catalytically active (due to a catalyst film) and an inert part of the device is measured by screen-printed thermopiles. When the absolute sensor temperature is modulated, the resulting signals depend on the catalyst's conversion behavior and are a characteristic for certain gas species. It could be shown that differentiation between gas components, e.g. alkanes and alkenes in a gas mixture is possible.
\end{abstract}

Key words: Thermoelectric gas sensors, exothermicity, catalysts conversion, differentiation, gas mixture

\section{Motivation and Background}

Distinguishing single components in a gas mixture is a main issue in gas sensing. Most gas sensor concepts based on mixed-potential or resistive metal-oxide principles respond towards several reducing gas components. Efforts were made in the past to improve the sensors' selectivity, for example by very special (but often not stable) electrode materials or complex multi sensor arrays [1]. In some studies, temperature modulation of such sensors to achieve a significant temperature dependent response for each single gas species is suggested. Unfortunately, these "fingerprints" depend on several parameters like electrode kinetics or electrochemical mechanisms [2-3].

In the present contribution, we use an indirect thermoelectric gas sensor. The direct thermoelectric principle measures gasdependent changes of the Seebeck-coefficient [4]. In general, indirect thermoelectric sensors measure a temperature difference $(\Delta T)$ between a catalytically active (catalyst coated) and an inert area [5]. Reducing gases in an oxygen-rich atmosphere get oxidized at the catalyst layer. Their exothermicity yields a temperature increase in this area. The sensor principle and its chemistry are very simple and well understood so that the sensors response only depends on the catalytic activity of the "active" area.

By modulating the working temperature, i.e. by changing the absolute sensor temperature and therefore the catalyst temperature, the sensor signal gives a measure of the catalyst light-off temperature and its catalytic conversion behavior (the measured thermovoltage is directly proportional to $\Delta T$ ). A "screening" of the temperature dependent catalytic activities gets possible. The sensor results depend on the gas species due to their individual conversion behavior. Therefore differentiation among various species is possible.

\section{Sensor Setup}

Sensors were built up in ceramic thick-film technology. As carrier we used either alumina (Rubalit 708S, CeramTec) or LTCC (951 Green Tape $^{\mathrm{TM}}$, DuPont) substrates. LTCC comprises a lower thermal conductivity [6] compared to alumina, which increases the sensor output, i.e. the sensitivity increases.

On the reverse side of the substrate, a Pt thickfilm heater (LPA88-11S, Heraeus) allows temperature adjustment and modulation. The heater is designed in a 4-wire setup so that an exact temperature control is possible by measuring its resistance in the hot area.

On the front side, the sensor comprises screenprinted Au/Pt-thermopiles, designed as sketched in Fig. 1 (Au: 5744R, DuPont, Pt: LPA88-11S, Heraeus). One finds a serial connection of $\mathrm{Pt}$ and $\mathrm{Au}$ feed lines in the heated zone of the sensor. The more thermopiles can be placed here, the higher is the signal output due to a temperature difference between a catalyst coated area (on the right hand side) and the inert area (on the left hand side). In our experiments, we used either serial connections 
of 9 or 15 thermocouples that form the thermopiles (details in [7]).

As the active component, i.e. for the catalyst coating, we used a porous alumina thick-film, which was activated with $1 \mathrm{wt} \% \mathrm{Pt}$.

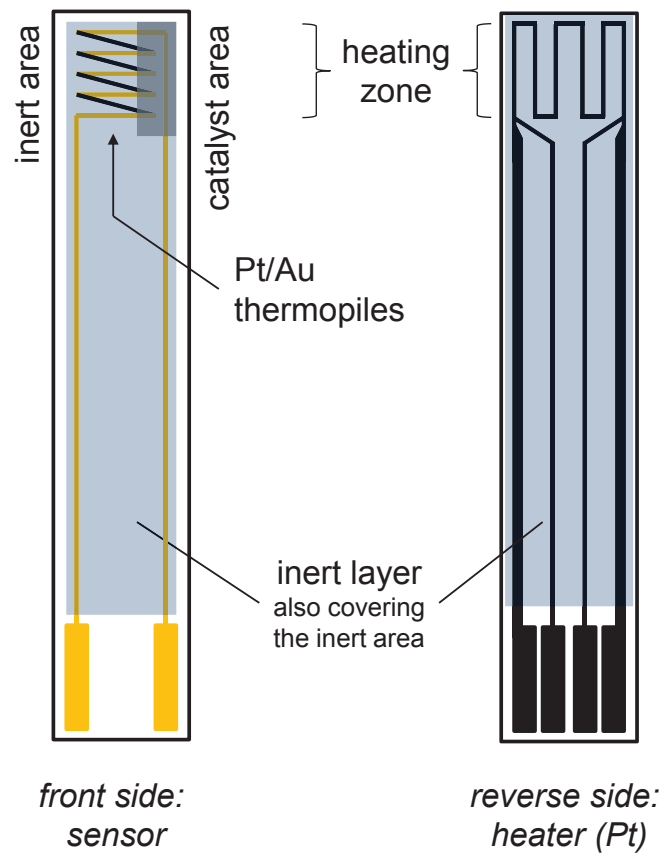

Fig. 1. Schematic sensor setup.

After wiring, we fixed the sensor in a simple stainless-steel housing for mounting it into a test bench (Fig. 2)

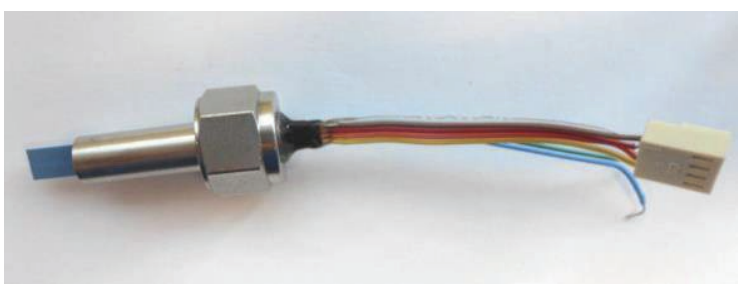

Fig. 2. Sensor, wired and housed, shown from the reverse side.

\section{Operation Strategy}

For temperature modulation, the outer heater feed-lines were connected to a waveform generator (HMF 2525, Rohde\&Schwarz HAMEG). Triangular voltage cycles were applied continuously (900 s per cycle). Current and voltage drop at the inner electrodes were measured by a digital-multimeter (Keithley 2700) for determining the heater resistance, which is a measure for the absolute sensor temperature $T$.

The sensor temperature $T$ is close to the catalyst temperature and is taken later as reference for evaluating the sensor results.

On the front side, a thermovoltage $\left(U_{\text {Sens }}\right)$ is measured during heater modulation. It represents the temperature difference between both areas of the thermopile structure. Exothermic reactions at the catalyst area lead to a temperature dependent characteristic signal for various gas components.

To visualize the light-off and conversion behavior over $T, U_{\text {sens }}$ must be corrected by the offset voltage ( $\left.U_{\text {offset }}\right)$. $U_{\text {offset }}$ means the sensor signal in the base gas atmosphere during heater modulation without exothermic reactions. $U_{\text {offset }}$ depends on various parameters like variations in the screen-printing process, heater structure or mounting position. One has to record these data once before the tests.

To achieve $\Delta T$ as a characteristic sensor result, the signal $\left(U_{\text {sens }}-U_{\text {offset }}\right)$ was also corrected by the temperature dependency of the Seebeck coefficient, which cannot be considered as linear in the entire temperature range. It amounts to $25.4 \mu \mathrm{V} / \mathrm{K} @ 1000{ }^{\circ} \mathrm{C}$ for Au/Pt. Data are presented in plots of $\Delta T$ vs. the sensor temperature $T$, which is here considered as the catalyst temperature.

All measurements were conducted in an oxidizing base gas atmosphere $\left(10 \% \mathrm{O}_{2}\right.$ in $\left.\mathrm{N}_{2}\right)$ with different reducing gas components or gas mixtures admixed to the base gas.

\section{Sensor Signal for Different Single Gases}

In a first test, single reducing gases were admixed to the base gas. As expected, the conversion behavior of the applied catalyst shows significant differences for various gas species. Fig. 3 shows representative results of an alumina-based sensor (nine thermocouples structure) for hydrogen $\left(\mathrm{H}_{2}\right)$, carbon monoxide (CO) and propane $\left(\mathrm{C}_{3} \mathrm{H}_{8}\right)$ admixture (5000 ppm each).

Both, the sensor signal $\left(U_{\text {Sens }}-U_{\text {offet }}\right)$ and the evaluated temperature difference between catalyst and inert area $\Delta T$ are shown in Fig. 3.

One can clearly distinguish between the gases as the sensor response appears in different temperature regions. Below $200{ }^{\circ} \mathrm{C}$, only $\mathrm{H}_{2}$ gets oxidized, $\mathrm{C}_{3} \mathrm{H}_{8}$ conversion starts above $400{ }^{\circ} \mathrm{C}$. In case of $\mathrm{CO}$ admixture a distinct lightoff is visible at $230{ }^{\circ} \mathrm{C}$, showing also a slight hysteresis in the cooling course. The shape of 
the conversion curve is characteristic for each gas species.

The sensor signal $\left(U_{\text {Sens }}-U_{\text {offset }}\right)$ reaches $500 \mu \mathrm{V}$ at $600{ }^{\circ} \mathrm{C}$ for $5000 \mathrm{ppm} \mathrm{H}_{2}$ or $\mathrm{C}_{3} \mathrm{H}_{8}$. This is equivalent to a temperature difference between the sensor areas of ca. $3 \mathrm{~K}$.

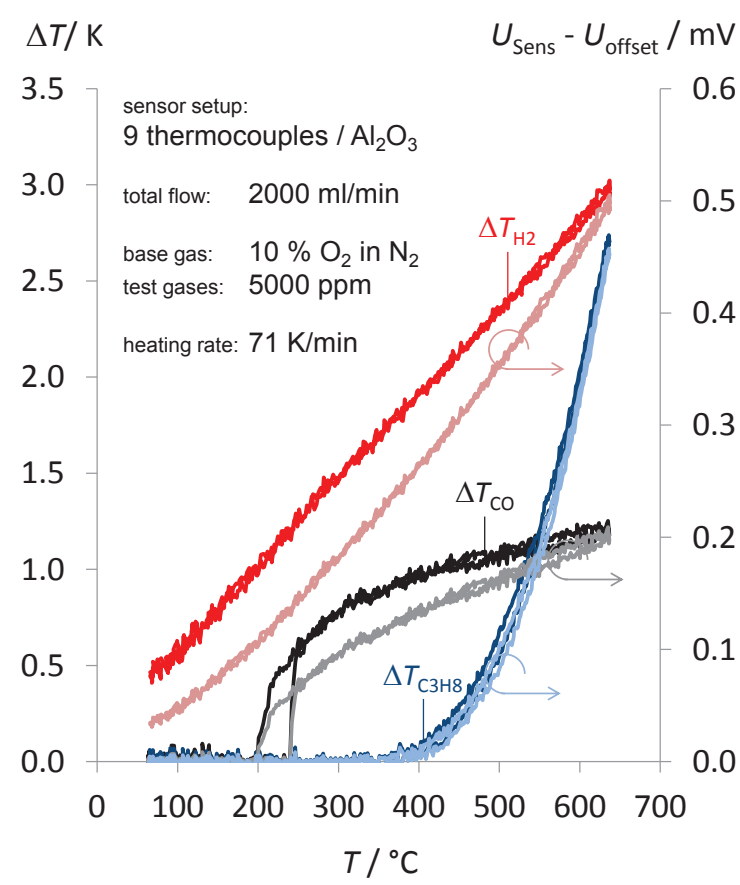

Fig. 3. Temperature difference and sensor signal vs. sensor temperature for different reducing gas components.

\section{Sensor Signal for Gas mixtures}

In another experiment, gas mixtures were examined. Here, we used an improved sensor setup (LTCC substrate with 15 thermocouples). Therefore, measurements of lower concentrations are possible (e.g. Fig. 4 and 5).

Ethine $\left(\mathrm{C}_{2} \mathrm{H}_{2}\right)$ and ethane $\left(\mathrm{C}_{2} \mathrm{H}_{6}\right)$ were selected as single gases (1000 ppm each) and as a mixture of both. The conversion behavior of ethane is very similar to that of propane already shown in Fig. 3. Ethine behaves like $\mathrm{CO}$ with a distinct light-off (at nearly $300{ }^{\circ} \mathrm{C}$ ) and slight hysteresis. The evaluated temperature signal $(\Delta T)$ is constant above $400{ }^{\circ} \mathrm{C}$ corresponding to a $100 \%$ conversion of this gas component on the catalyst.

A mixture of both gases should lead to a superposition of the output of each single gas. Fig. 4 clearly confirms this result. Similar results could be achieved with other alkanes mixed with unsaturated hydrocarbons.

\section{$\Delta T / K$}

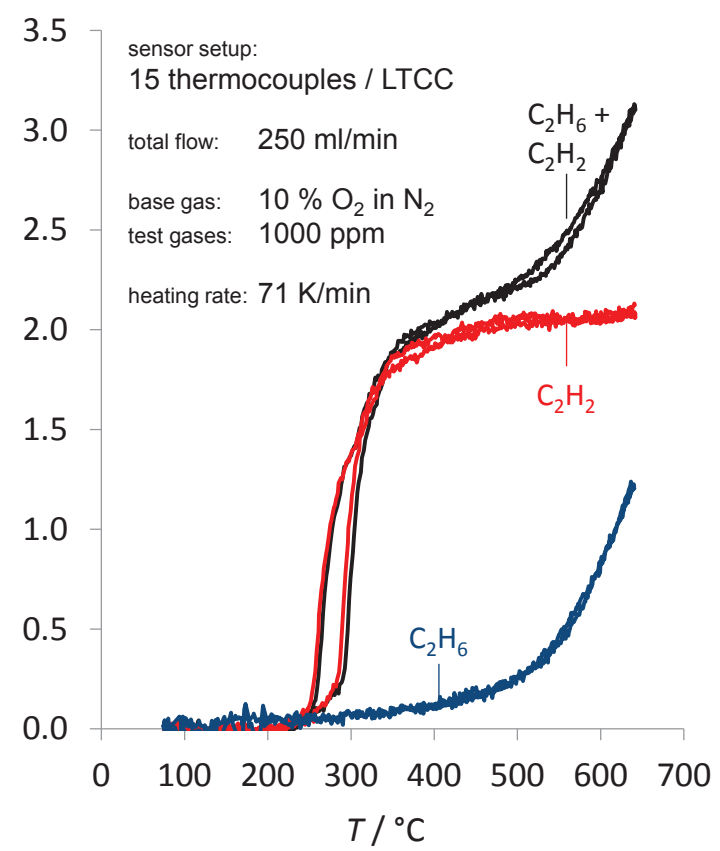

Fig. 4. Temperature difference $(\Delta T)$ obtained from the sensor signal vs. sensor temperature for ethine and ethane (single gases and mixture). Compared to Fig. 3, LTCC with a low thermal conductivity was used as substrate material and 15 thermocouples formed the thermopile

These findings could lead to a sensor application for multi-gas detection, at least to distinguish between alkanes and unsaturated hydrocarbons.

However, also some issues with respect to catalysis need to be considered. In some cases, conversion is blocked by gas species with a higher light-off temperature. Then, differentiation between these components is difficult.

This is demonstrated in the following experiment. Here, propene and ethine were measured as single gases and also as gas mixtures (parameters and concentrations like before). If one compares the sensor output signal (again transformed into a temperature difference) of a mixture and the calculated superposition of the signals from single gas components, it becomes clear that ethine hinders the propene conversion at the catalyst. The light-off of propene as single gas is measured at $200{ }^{\circ} \mathrm{C}$, in the mixture propene conversions starts with the ethine light-off at $300^{\circ} \mathrm{C}$. 


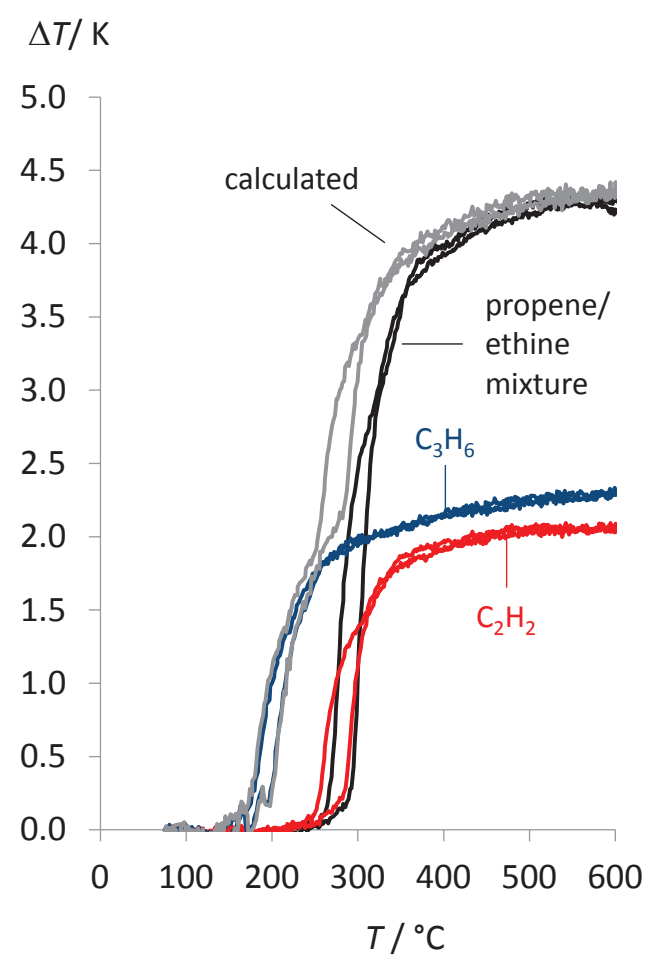

Fig. 5. Temperature differences $(\Delta T)$ vs. sensor temperature for ethine and propene (single gases and mixture).

Similar effects can be seen in case of mixtures with $\mathrm{CO}$. For example, $\mathrm{CO}$ hinders the $\mathrm{H}_{2}$ conversion. This is known from literature [8].

\section{Conclusion}

All in all, the temperature modulation strategy offers an interesting benefit for multi-gas analysis. In combination with the thermoelectric sensor principle, its simple setup / chemistry and its robust design (use of high temperature stable materials), many applications come into sight such as control and diagnosis of combustion processes or for applications in the field of safety devices.

Furthermore, such a setup could be a powerful tool for catalyst research and development.

\section{References}

[1] R. Moos, K. Sahner, M. Fleischer, U. Guth, N. Barsan, U. Weimar, Solid State Gas Sensor Research in Germany - a Status Report, Sensors 9, 4323 - 4365 (2009), doi: 10.3390/s90604323

[2] H. Kohler, J. Röber, N. Link, I. Bouzid, New application of tin oxide gas sensors I. Molecular identification by cyclic variation of the working temperature and numerical analysis of the signals, Sensors and Actuators B 61, 163-169 (1999), doi: 10.1016/s0925-4005(99)00286-5

[3] A. Gramm, A. Schütze, High performance vapor identification with a two sensor array using temperature cycling and pattern classification, Sensors and Actuators B 95, 58-65 (2003), doi: 10.1016/s0925-4005(03)00404-0

[4] F. Rettig, R. Moos, Direct thermoelectric gas sensors: Design aspects and first gas sensors, Sensors and Actuators B 123, 413 - 419 (2007), doi: 10.1016/j.snb.2006.09.002

[5] S. Wiegärtner et al., Thermoelektrischer Kohlenwasserstoffsensor in Dickschichttechnik zur On-Board-Diagnose eines Diesel-OxidationsKatalysators, Sensoren und Messsysteme 2014, 3.6.-4.6.2014, Nürnberg, ISBN 978-3-8007-36225

[6] J. Kita, A. Engelbrecht, F. Schubert, A. Groß, F. Rettig, R. Moos, Some practical points to consider with respect to thermal conductivity and electrical resistivity of ceramic substrates for high-temperature gas sensors, Sensors and Actuators B: Chemical, in press, doi: 10.1016/j.snb.2015.01.041

[7] S. Wiegärtner, G. Hagen, J. Kita, W. Reitmeier, M. Hien, P. Grass, R. Moos, Thermoelectric Hydrocarbon Sensor in Thick-film Technology for On-Board-Diagnostics of a Diesel Oxidation Catalyst, Sensors and Actuators B, accepted, doi: 10.1016/j.snb.2015.02.083

[8] M. Nishibori, W. Shin, K. Tajima, L. Houlet, N. Izu, T. Itoh, S. Tsubota, I. Matsubara, Thermoelectric Gas Sensor using Au Loaded Titania CO Oxidation Catalyst, Journal of the Ceramic Society of Japan 115, 37-41 (2007), doi: 10.2109/jcersj.115.37 